\title{
MICROFLUIDIC PUMPING BASED ON TRAVELING-WAVE DIELECTROPHORESIS
}

\author{
Dong Liu' ${ }^{1}$ and Suresh V. Garimella ${ }^{2}$ \\ ${ }^{1}$ Department of Mechanical Engineering, University of Houston, Houston, Texas, \\ USA \\ ${ }^{2}$ School of Mechanical Engineering and Birck Nanotechnology Center, Purdue \\ University, West Lafayette, Indiana, USA
}

\begin{abstract}
This article presents a microfluidic pumping approach using traveling-wave dielectrophoresis (twDEP) of microparticles. With this approach, the flow is generated directly in the microfluidic devices by inducing strong electromechanical effects in the fluid using integrated microelectrodes. The fluidic driving mechanisms due to the particle-fluid and particle-particle interactions under twDEP are analyzed, and the induced flow field is obtained from numerical simulations. Experimental measurements of the flow velocity in a prototype DEP micropumping device show satisfactory agreement with the numerical predications. Results from this work indicate that the DEP-induced micropumping scheme holds great promise for devising versatile, self-contained microscale fluidic delivery systems.
\end{abstract}

KEY WORDS: dielectrophoresis, traveling-wave field, particle-fluid interaction, micropumping, microfluidics

\section{INTRODUCTION}

Novel microfluidic devices are being developed for various applications, including drug delivery [1], rapid chemical synthesis [2], biological diagnostics [3], and electronics cooling [4]. The ability to actuate and control fluid in small amounts with high precision and flexibility is critical to the success of microfluidic operations. Conventional pressuredriven pumping methods are inadequate in accommodating these requirements mainly due to the large pressure head needed; moreover, the use of an external pump in a microfluidic system defeats the purpose of miniaturization. Alternative solutions have been sought and a variety of innovative micropumping concepts have been proposed in the literature $[5,6]$. One particularly attractive scheme is to generate the required flow directly in the microfluidic devices by inducing strong electromechanical forces in the fluid through electrokinetic effects. Based on the origin of the electromechanical forces, electrokinetic

Received 9 June 2008; accepted 13 March 2009.

The authors acknowledge assistance from Vaibhav Bahadur and Brian Iverson of Purdue University with different aspects of this work.

An earlier version of this work was presented at the Intersociety Conference on Thermal and Thermomechanical Phenomena in Electronic Systems (ITHERM), Orlando, FL, 2008.

Address correspondence to Suresh V. Garimella, School of Mechanical Engineering and Birck Nanotechnology Center, 585 Purdue Mall, Purdue University, West Lafayette, IN 47907-2088. E-mail: sureshg@purdue.edu 


\begin{tabular}{|llll|}
\hline \multicolumn{2}{|c|}{ NOMENCLATURE } \\
$A$ & area & $V$ & velocity \\
$a$ & particle radius & & \\
$d_{1}$ & electrode width & Greek Symbols \\
$d_{2}$ & spacing between neighboring & $\varepsilon$ & dielectric permittivity \\
& electrodes & $\mu$ & viscosity \\
$E$ & electric field & $\rho$ & mass density \\
$F$ & dielectrophoretic force & $\sigma$ & electrical conductivity \\
$f$ & frequency of the applied electrical & $\varphi$ & phase angle \\
& signal & $\omega$ & frequency \\
$f_{C M}$ & Clausius-Mossotti factor & & \\
$L$ & interparticle distance & Subscripts \\
$m$ & mass & $f$ & fluid \\
$p$ & dipole moment & $m$ & medium \\
$t$ & time & $p$ & particle \\
$u$ & velocity & & \\
\cline { 2 - 4 } & & & \\
\hline
\end{tabular}

micropumps can be classified as electrohydrodynamic (EHD) [7-9], electroosmotic (EO) [10], and AC electroosmotic (ACEO) [11, 12], among others. The common feature of these micropumps is to actuate the liquid via an induced body force directly exerted on the fluid element. Recently, more complex fluids, such as colloidal suspensions containing a second phase (vapor bubbles, solid/soft particles, or immiscible liquid droplets) have received attention in microfluidics research and applications. Examples include separation/concentration of biological cells in micro-total-analysis systems ( $\mu$ TAS) [13] and application of nanofluids in advanced cooling systems [14]. Due to the presence of the second phase in the fluid, another important electrokinetic effect, dielectrophoresis (DEP), can be exploited to generate effective microfluidic pumping upon the application of an external electric field.

Dielectrophoresis is the motion of small particles in colloidal suspensions when exposed to nonuniform electric fields, arising from the interaction of the induced dipole on the particle with the applied field [15]. Dielectrophoresis has been employed extensively as a powerful tool for manipulating particles in biological research, such as in separation [16], trapping [17], sorting [18], and translation [19] of cells, viruses, proteins, and DNA. However, DEP research to date has focused on controlling the electromechanical response of the solid particles while largely neglecting the hydrodynamic interactions between the particles and the surrounding fluid, i.e., the motion of the surrounding fluid induced by drag from the dielectrophoretic particle motion due to viscous effects. In spite of the advances in colloid science and electromechanics [20-22], a gap still persists in the application of advances in the science of particle dynamics and low-Reynolds-number hydrodynamics to the DEP technique. This gap must be bridged to facilitate the implementation of DEP in a broader range of applications. In particular, the potential of traveling-wave DEP (twDEP) as an effective means for microfluidic flow actuation has not yet been explored.

The present work aims to develop an electrokinetic micropumping concept that capitalizes on the DEP-induced hydrodynamic interaction between small particles and the surrounding fluid and to utilize this concept to devise self-contained microfluidic delivery systems. In this article, an analysis of dielectrophoresis and the DEP force is first presented as a basis for the discussion of electromechanical transport. Fundamental aspects of the hydrodynamic interaction between the particles and the surrounding fluid are then 
discussed and detailed information on the DEP-induced flow field is obtained from numerical analysis. The development of a prototype DEP micropump and experimental characterization of the DEP-induced flow velocity are then reported. Finally, the potential for actuating nanofluids with the DEP-induced micropumping technique is discussed.

\section{THEORY AND ANALYSIS}

Redistribution of the electrical charges in a dielectric particle suspended in a fluid medium upon exposure to an applied external electric field establishes net charges at the interface between the particle and the fluid and forms an induced dipole across the particle. The induced dipole tends to align with the applied field. The induced dipole moment, $\vec{p}$, and the dielectrophoretic force, $\vec{F}$, are given by

$$
\begin{gathered}
\vec{p}=4 \pi a^{3} \varepsilon_{m}\left(\frac{\varepsilon_{p}-\varepsilon_{m}}{\varepsilon_{p}+2 \varepsilon_{m}}\right) \vec{E} \\
\vec{F}=(\vec{p} \cdot \nabla) \vec{E}=2 \pi a^{3} \varepsilon_{m}\left(\frac{\varepsilon_{p}-\varepsilon_{m}}{\varepsilon_{p}+2 \varepsilon_{m}}\right) \nabla \vec{E}^{2}
\end{gathered}
$$

in which $a$ is the radius of the particle, $\vec{E}$ is the applied electric field vector, and $\varepsilon_{\mathrm{m}}$ and $\varepsilon_{p}$ are the dielectric permittivity of the fluid medium and the particle, respectively. If the applied field is nonuniform $(\nabla \vec{E} \neq 0)$, the particle will experience a net force and move by the process of dielectrophoresis [23, 24]. DEP takes place in both direct current (DC) and alternating current (AC) electric fields. Sustained particle motion only occurs in AC DEP with the appropriate driving frequencies (in particular, in traveling-wave DEP), for which case, the permittivity in Eq. (2) is replaced by the frequency-related counterpart,

$$
\tilde{\varepsilon}=\varepsilon-i \frac{\sigma}{\omega}
$$

in which $\varepsilon$ and $\sigma$ are the permittivity and electrical conductivity of the dielectric materials, and $\omega$ is the angular frequency of the electric field.

While the particle travels via DEP in a surrounding fluid, it suffers a retarding drag force if the fluid is either moving slower than the particle or otherwise stationary. The fluid surrounding the particle is in turn dragged by viscous effects to accelerate in the same direction as the particle. The momentum exchange between the particle and the fluid reduces the velocity lag between the phases and eventually leads to an equilibrium state. A steady flow field is then established around the particle in the fluid as a result of this hydrodynamic interaction. In a particle suspension, a large collection of particles is present and the particles further interact hydrodynamically with neighbors. Consequently, the induced flow field is intensified and an appreciable net flow is produced by the collective pumping action. This is the basic electromechanical transport process underlying the DEP-induced microfluidic pumping technique investigated here.

\section{Traveling-Wave Dielectrophoresis (twDEP)}

The AC dielectrophoretic force on the particle is expressed using the frequency-dependent permittivity as [25] 


$$
\vec{F}=2 \pi a^{3} \varepsilon_{m}\left(\frac{\tilde{\varepsilon}_{p}-\tilde{\varepsilon}_{m}}{\tilde{\varepsilon}_{p}+2 \tilde{\varepsilon}_{m}}\right) \nabla \vec{E}^{2}
$$

The complex relative permittivity is also referred to as the Clausius-Mossotti factor, $f_{C M}$,

$$
\tilde{f}_{C M}=\frac{\tilde{\varepsilon}_{p}-\tilde{\varepsilon}_{m}}{\tilde{\varepsilon}_{p}+2 \tilde{\varepsilon}_{m}}
$$

Assuming the electric field varies with a single angular frequency $\omega$, the timeaveraged dielectrophoretic force can be computed as [26]

$$
\left\langle\vec{F}_{D E P}\right\rangle=\pi a^{3} \varepsilon_{m} \operatorname{Re}\left[f_{C M}\right] \nabla|\vec{E}|^{2}+2 \pi a^{3} \varepsilon_{m} \operatorname{Im}\left[f_{C M}\right]\left(E_{x}^{2} \nabla \varphi_{x}+E_{y}^{2} \nabla \varphi_{y}+E_{z}^{2} \nabla \varphi_{z}\right)
$$

where $\operatorname{Re}\left[f_{C M}\right]$ and $\operatorname{Im}\left[f_{C M}\right]$ denote the real and imaginary parts of $f_{C M}$, and $E_{x}, E_{y}$, and $E_{z}$ are components of the electric field vector; $\varphi_{x}, \varphi_{y}$, and $\varphi_{z}$ are the phase angles if the electric field is spatially phase-shifted. It is noted that the DEP force depends on the spatial nonuniformities in both the field strength $\left(\nabla|\vec{E}|^{2}\right)$ and the phase $(\nabla \varphi)$. In fact, the first term on the right-hand side of Eq. (6) determines the alignment of the DEP force with respect to the maxima/minima of the electric field and is the regular DEP force component in DC DEP. The second term on the right-hand side of Eq. (6). only appears if the electric field has a spatially varying phase, such as in a traveling-wave field, and therefore is the traveling-wave DEP (twDEP) force component. It is noted that the dipole approximation used in deriving Eq (6). is no longer valid in regions very close to the electrodes where the particle size becomes comparable to the characteristic length scale of the field nonuniformity. Because the DEP micropumping of interest occurs at a sufficient distance from the electrode plane, Eq. (6). is employed for its clear physical meaning as well as its ease of implementation over other methods in the literature [27].

The alignment of the DEP force with the applied field is contingent upon the Clausius-Mossotti factor $f_{C M}$, which is frequency dependent. Figure 1 illustrates the real and imaginary parts of $f_{C M}$ as a function of the frequency of the applied field for polystyrene particles suspended in water. Clearly, $\operatorname{Re}\left[f_{C M}\right]$ is positive in the lowfrequency range $(f<1 \mathrm{kHz})$ in which the particles are more polarizable than the surrounding fluid and crosses over to negative values as the frequency increases $(f>100 \mathrm{kHz})$ and the particles become less polarizable than the fluid. If $\operatorname{Re}\left[f_{C M}\right]>0$, the regular DEP force component aligns favorably with the field strength gradient, as indicated by Eq. (6). As a result, the particles move toward the maxima of the electric field, which are usually located at the edges of the electrodes that are used to generate the electric field, and positive DEP occurs. In the opposite situation, a negative $\operatorname{Re}\left[f_{C M}\right]$ brings about negative DEP where the particles move away from the maxima of the electric field, distancing themselves from the electrodes. $\operatorname{Im}\left[f_{C M}\right]$ vanishes at both extremes of the frequency spectrum but assumes non-zero values in the mid-range around the crossover frequency. When $\operatorname{Im}\left[f_{C M}\right]$ is not trivial, the resulting twDEP force in Eq. (6) propels the particles along or against the propagating traveling-wave field depending on the sign of $\operatorname{Im}\left[f_{C M}\right]$. The twDEP force is generally oriented in parallel to the electrode plane. However, in practice, twDEP does not occur in isolation without the companion negative DEP, because the particles must be levitated from the electrode surface. As such, the criteria for effective twDEP are 


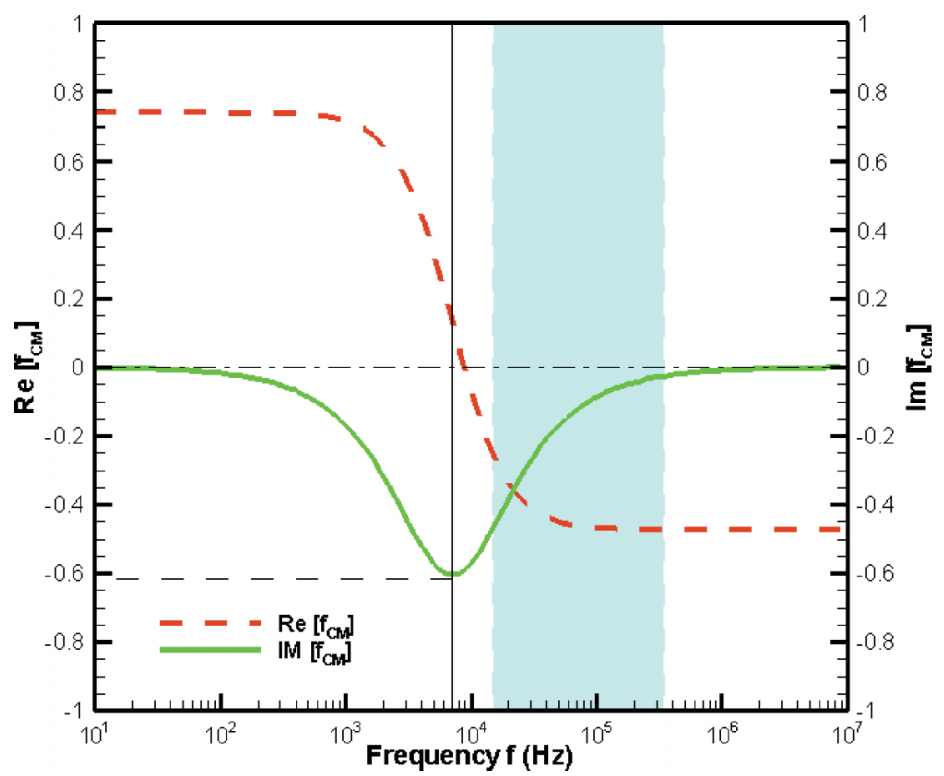

Figure 1 Frequency dependence of the Clausius-Mossotti factor $f_{C M}$. The real part is shown by the broken line and the imaginary by the solid line. At low frequencies, positive DEP occurs and particles are attracted onto the electrodes; at high-frequency range, negative DEP occurs and particles are repelled and freed from the electrode. The shaded area indicates the effective frequency range for twDEP.

$\operatorname{Re}\left[f_{C M}\right]<0$ and $\operatorname{Im}\left[f_{C M}\right] \neq 0$, which are designated by the shaded area on the frequency spectrum in Figure 1.

\section{Electric Field}

The electric field needed for twDEP is often generated by applying a travelingwave voltage signal to specially designed electrode arrays. In the present study, threephase, planar parallel electrodes are fabricated on the bottom surface of the flow channel. As shown in Figure 2, the electrodes are $9 \mathrm{~mm}$ long and have width and uniform spacing of $d_{1}=20 \mu \mathrm{m}$ and $\mathrm{d}_{2}=180 \mu \mathrm{m}$, respectively. The fluid and particles are assumed to be homogeneous linear dielectric materials, so that the electric field in the particle suspension in the flow channel can be solved using Laplace's equation. An insulating layer of Parylene C (thickness $500 \mathrm{~nm}$ ) present on the electrode array is neglected in the electric field model. Past analytical solutions include approaches using Fourier series [26], Green's theorem [28], and the halfplane Green's function [29], and semi-analytical methods include the charge density method [30] and Green's function for a line source with conformal mapping [31]. All these solution approaches have used a linear approximation of the electric potential in the gap between consecutive electrodes as the boundary condition. It will be shown that this is not a good assumption and can cause large errors in the analysis. The calculation can be improved by employing numerical methods [32]. Hence, a commercial software package, FLUENT, is used here to simulate the electrical field by solving the scalar transport equations [33]. 

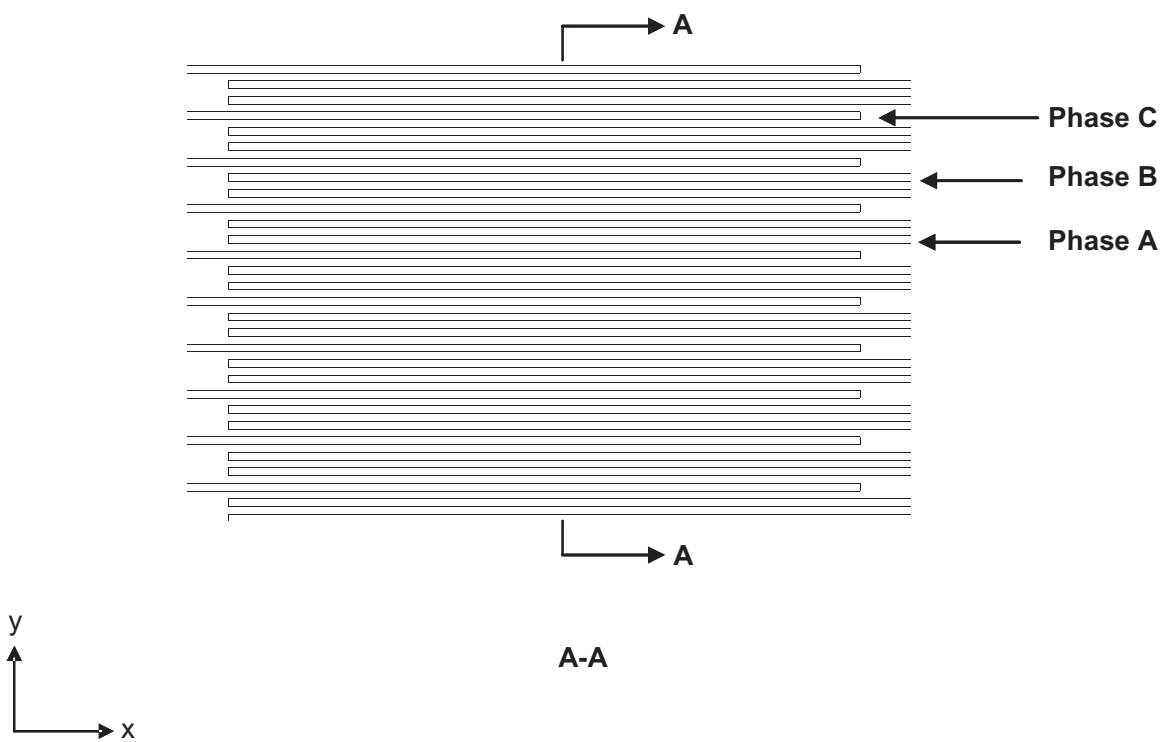

A-A

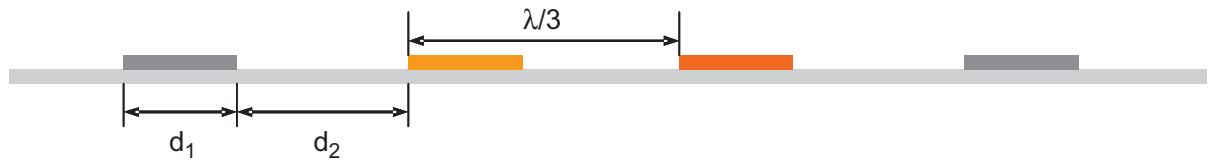

Figure 2 Three-phase planar microelectrode array. The electrode width is $d_{1}=20 \mu \mathrm{m}$ and the spacing between electrodes is $d_{2}=180 \mu \mathrm{m}$. The wavelength of the applied voltage signal is $\lambda\left[=3\left(d_{1}+d_{2}\right)\right]$.

The electric potential for an AC field of angular frequency $\omega$ is [26]

$$
\phi(\vec{x}, t)=\phi_{1} \cos (\omega t)+\phi_{2} \sin (\omega t)
$$

where both $\phi_{1}(x, y)$ and $\phi_{2}(x, y)$ satisfy Laplace's equation $\nabla^{2} \phi_{i}=0(i=1,2)$. In the three-phase traveling-wave field, the voltages on consecutive electrodes are phase-shifted by $120^{\circ}$, such that $\phi_{2}(x, y)=\phi_{1}(x-\lambda / 3, y)$, where the wavelength $\lambda=3\left(d_{1}+d_{2}\right)$. After solving for the electric potential, the electric field is obtained from $\vec{E}(\vec{x}, t)=-\nabla \phi=\vec{E}_{1}(x, y) \cos (\omega t)+\vec{E}_{2}(x, y) \sin (\omega t)$, where $\quad \vec{E}_{1}(x, y)=-\nabla \phi_{1} \quad$ and $\vec{E}_{2}(x, y)=-\nabla \phi_{2}$.

For the electrode array used in the present study, the length $(9 \mathrm{~mm})$ along the transverse direction (length of the electrodes) can be considered infinite relative to the other two dimensions, as shown in Figure 2, so that the electrode array is treated as a two-dimensional system. The computational domain and the boundary conditions are illustrated in Figure 3. Due to periodicity in the electric field, only a distance along the electrodes of one wavelength is modeled, covering three electrodes and their gaps. Periodic boundary conditions are imposed at the vertical edges of the computational domain shown. On the top surface, which is located at a distance of $h=200 \mu \mathrm{m}$ from the electrode array, a Neumann condition $\left(\frac{\partial \phi}{\partial n}=0\right)$ is assumed because insulating Pyrex glass (dielectric constant, $\varepsilon_{r}=4.8$ ) is used in the experiments to enclose the flow channel, which is filled with water $\left(\varepsilon_{r}=78.4\right)$. On the bottom surface, the electrodes 


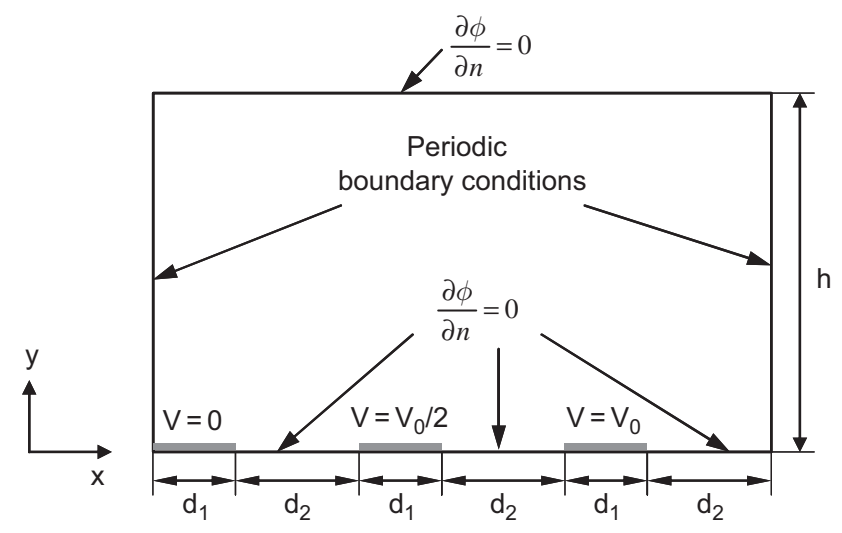

Figure 3 A schematic diagram of the computational domain for the electric field. Boundary conditions are shown for all surfaces. The same configuration is also used in computing the DEP-induced flow field.

are represented by sections with specified values of voltages. In the gap regions between neighboring electrodes, the more physically representative Neumann condition is specified for the electric field instead of using a linear approximation.

Numerical results for the electric potential and the electric field are shown in Figure 4. Figure 4(a) shows that the electric potential decays rapidly with increasing distance from the electrode surface. Because the density of the field lines is proportional to the strength of the electric field, Figure 4(b) shows clearly that the field maxima are located near the edges of the electrodes. Interestingly, the second-phase electrode does not appear to have an influence in Figure 4(b) because most field lines bypass this electrode and connect directly between the first- and third-phase electrodes. However, the second-phase electrode is indispensable because otherwise the phase-angle term would vanish in Eq. (6) and no useful traveling-wave field would be generated for the twDEP application. Figure 4(c) illustrates the exact solution for the electric potential at the electrode surface, which exhibits significant deviation from the first-order linear approximation often made in past studies in the literature.

\section{DEP Forces}

Once the traveling-wave electric field is solved, the time-averaged DEP force can be recast in the following form [28, 34]:

$$
\begin{aligned}
\left\langle\vec{F}_{D E P}\right\rangle= & \pi a^{3} \varepsilon_{m} \operatorname{Re}\left[f_{C M}\right] \vec{\nabla}\left(E_{x 1}^{2}+E_{x 2}^{2}+E_{y 1}^{2}+E_{y 2}^{2}\right) \\
& +2 \pi a^{3} \varepsilon_{m} \operatorname{Im}\left[f_{C M}\right]\left(E_{x 1} \vec{\nabla} E_{x 2}-E_{x 2} \vec{\nabla} E_{x 1}+E_{y 1} \vec{\nabla} E_{y 2}-E_{y 2} \vec{\nabla} E_{y 1}\right)
\end{aligned}
$$

in which $E_{x 1}$ and $E_{y 1}$ correspond to $\phi_{1}$, and $E_{x 2}$ and $E_{y 2}$ correspond to $\phi_{2}$. As will be seen, the first term, which is the regular DEP force component, controls the vertical motion of the particle, and the second term, which is the traveling-wave DEP force component, is responsible for particle motion in the flow direction. These two force components together give rise to the DEP-based microfluidic pumping considered in this work. 


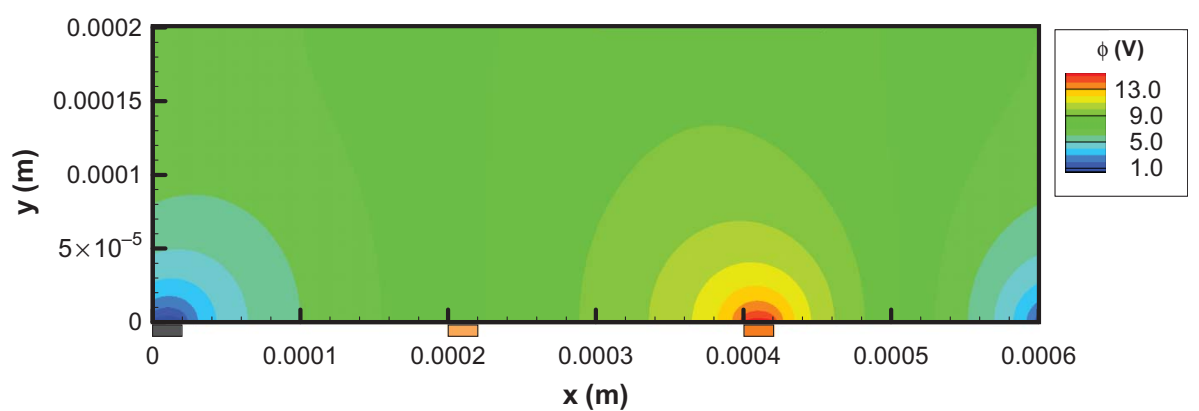

(a)

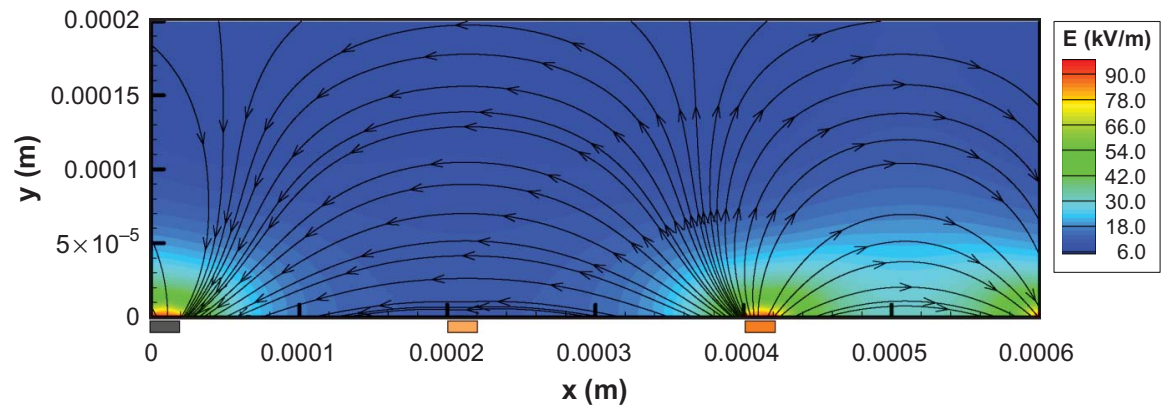

(b)

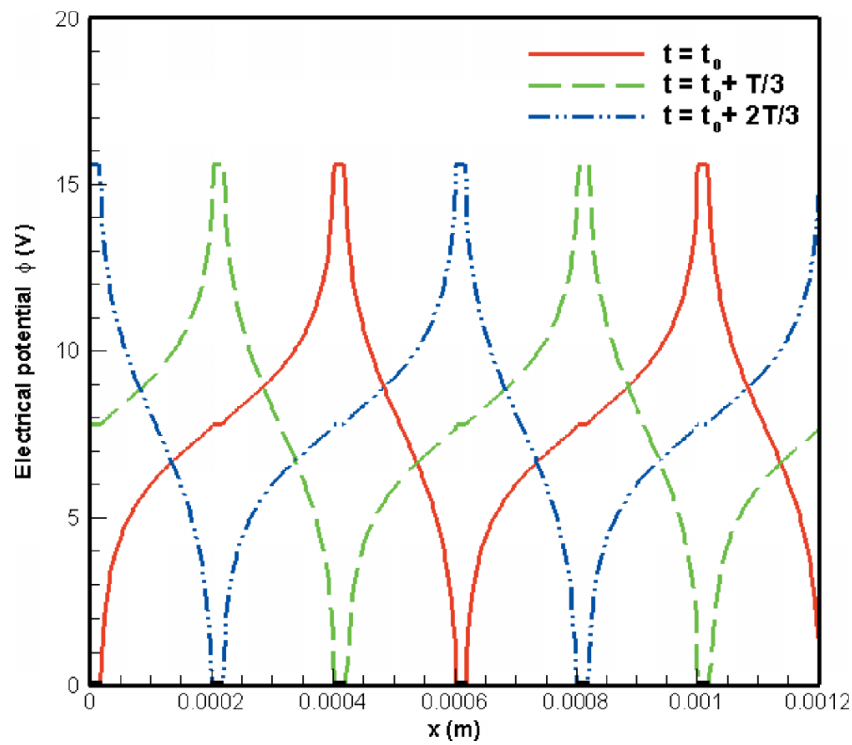

(c)

Figure 4 Solutions of the electric field calculated from the numerical model $\left(V_{0}=15.6 \mathrm{~V}\right)$ : (a) The contour of the electric potential (color-coded) exhibits a rapid decay in the far-field region (the positions of the electrodes are indicated by three colored bars below the $x$-axis; the same notation applies for all figures that follow); (b) the contour of the electric field (color-coded) shows maxima near the electrodes, which is also indicated by the field streamlines; and (c) the electric potential at the electrode surface deviates dramatically from the linear approximation often made in past analyses. 


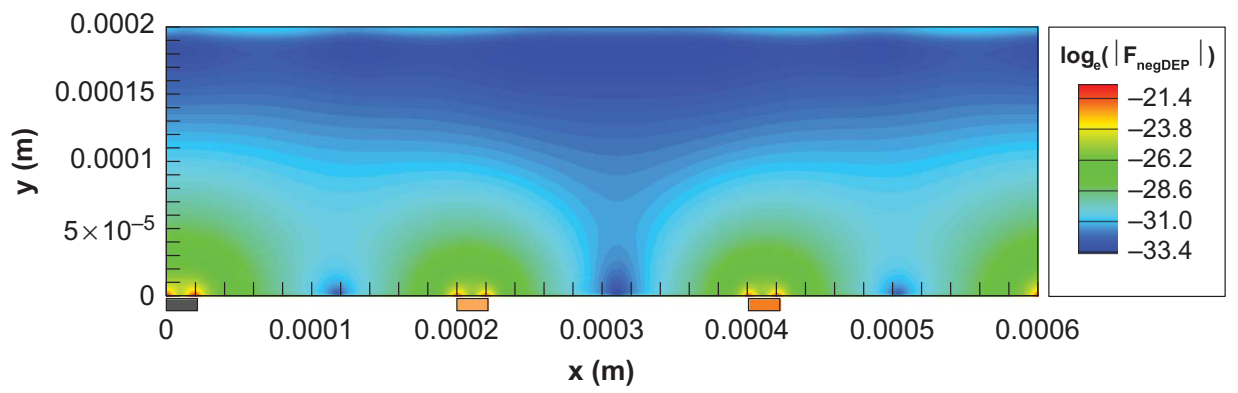

(a)

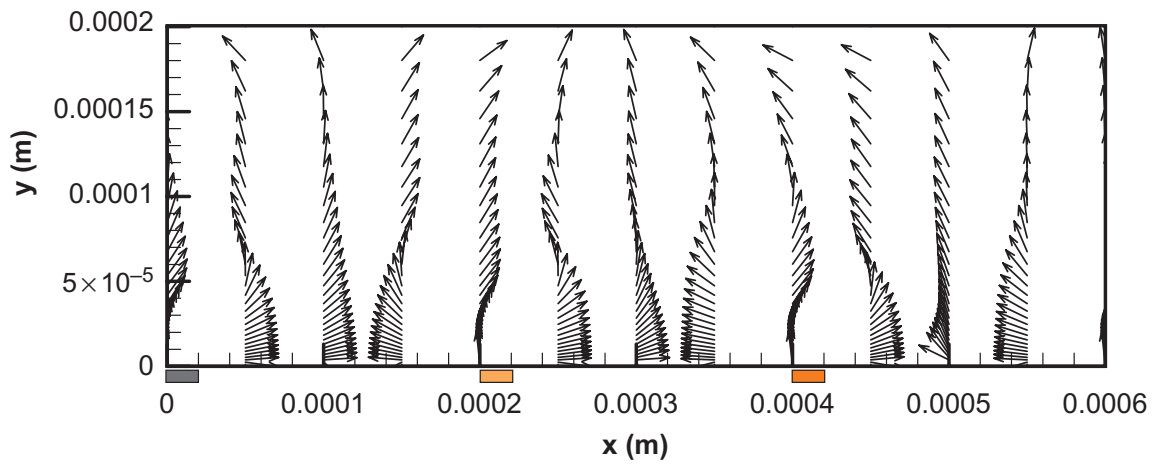

(b)

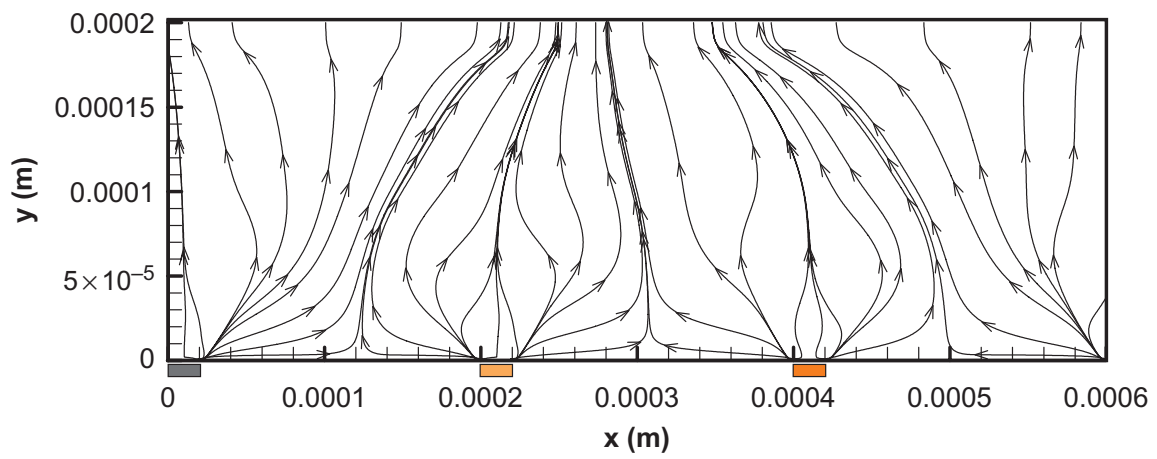

(c)

Figure 5 Negative DEP force for $\operatorname{Re}\left[f_{C M}\right]=-0.5$ and $\operatorname{Im}\left[f_{C M}\right]=0$ : (a) The magnitude of the DEP force (in units of $N$ ) is largely uniform at the same height except for regions near the electrodes (plotted in logarithmic color scale); (b) the DEP force points upwards (the plotted force vectors only indicate the direction, not the magnitude); and (c) particles would be levitated above the electrode surface by the negative DEP force as indicated by the streamlines.

Negative DEP force Negative DEP is required for twDEP to occur. Figure 5(a) shows contours of the DEP force calculated for a pure negative DEP case, corresponding to $\operatorname{Re}\left[f_{C M}\right]=-0.5$ and $\operatorname{Im}\left[f_{C M}\right]=0$, which shows that the strength of the DEP force is largely uniform except for regions near the electrodes. Figure 5(b) indicates that the DEP force points outwards from the 
electrode edge against the gradient of the electric field. The streamlines in Figure 5(c) show more clearly that a particle suspended in the fluid tends to be levitated away from the electrode surface.

Traveling-wave DEP force In a three-phase traveling-wave field, the spatially varying phase makes the horizontal motion of the particle possible. Figure 6

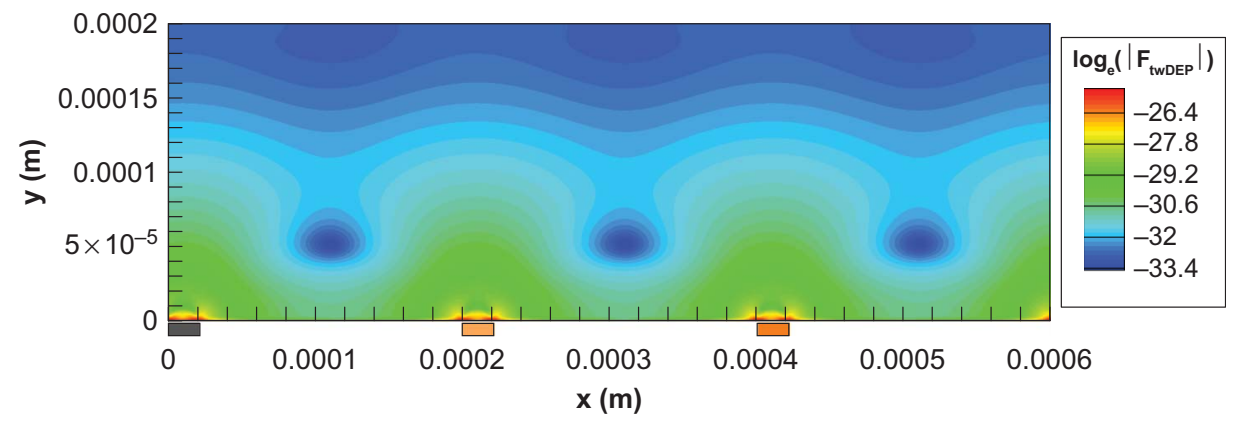

(a)

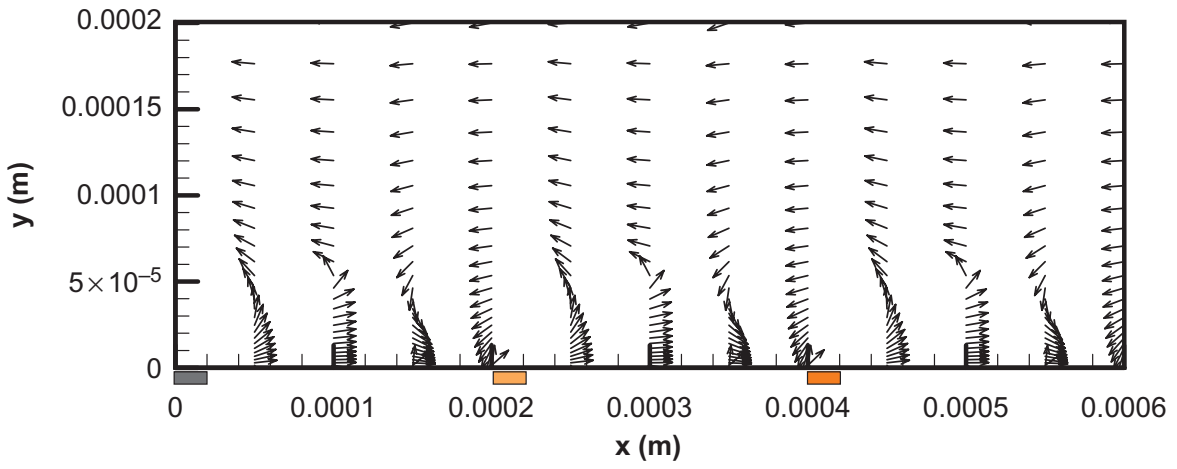

(b)

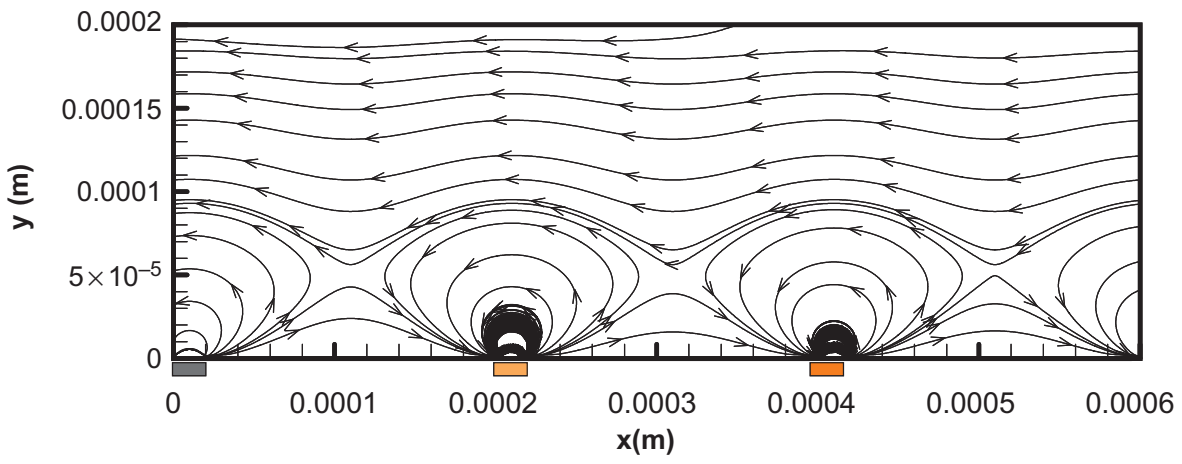

(c)

Figure 6 Traveling-wave DEP force for $\operatorname{Re}\left[f_{C M}\right]=0$ and $\operatorname{Im}\left[f_{C M}\right]=-0.4$ : (a) the twDEP force (in units of $N$ ) exhibits periodic patterns due to its traveling-wave nature (plotted in logarithmic color scale); (b) the twDEP force component exhibits the same periodic patterns and is mostly horizontal by a certain height above the electrode surface (the plotted force vectors only indicate the direction, not the magnitude); and (c) particles would translate horizontally away from the electrode surface and circulate between the electrodes as indicated by the streamlines. 
illustrates the pure twDEP force and the streamlines corresponding to $\operatorname{Re}\left[f_{C M}\right]=0$ and $\operatorname{Im}\left[f_{C M}\right]=-0.4$. Figure 6 (a) shows a periodic profile for the DEP force strength, in contrast to that in the case of negative DEP (Figure 5(a)), which is consistent with the traveling-wave nature of the field. Figure 6(b) illustrates that at some height above the electrode surface, the twDEP force becomes nearly uniform in magnitude and acts against the propagating traveling wave in the horizontal direction. At lower heights, trajectories of the particle would no longer conform to translational motion and vortices can be found between the electrodes as evident from the streamline plot in Figure 6(c).

It should be noted that the electric field and the DEP force field obtained from Eqs. (7) and (8) are only approximate, because the voltage signals applied to the electrodes are not truly sinusoidal traveling waves, as shown in Figure 4(c). Therefore, the foregoing treatment captures only the first-order effects of the imposed electric field; however, it represents a reasonable approximation, as will be seen from the agreement between the velocity field obtained from this simulation and the experimental measurement.

\section{Particle-Fluid Interactions in DEP-Induced Flows}

Particle-fluid hydrodynamic interactions are central to the DEP-induced micropumping concept proposed here. A particle experiences a variety of external forces as it travels in the surrounding fluid. The single particle dynamics can be described by the Langevin equation [35]

$$
m \frac{d^{2} \vec{r}}{d t^{2}}=\vec{F}_{G}+\vec{F}_{D E P}+\vec{F}_{v}+\vec{R}(t)+\sum \vec{F}_{\text {add }}^{i, j}
$$

in which the gravitational force is $\vec{F}_{G}=\frac{4}{3} \pi a^{3}\left(\rho_{p}-\rho_{f}\right) \vec{g}$, the time-averaged DEP force $\vec{F}_{D E P}$ is given by Eq. (6), the viscous drag force is described by Stokes' drag law $\vec{F}_{v}=-6 \pi \mu_{f} a\left(\vec{u}_{m}-\vec{u}_{p}\right)$, and the random Brownian force is $\vec{R}(t)$ for which the diffusion coefficient is $D_{B}=k_{B} T /\left(6 \pi \mu_{f} a\right)$. The additional terms $\vec{F}_{a d d}{ }^{i, j}$ arise in a suspension of multiple particles and account for the electrical interactions between neighboring particles [36]. In the experiments for the present work, polystyrene particles $\left(\rho_{p}=1050 \mathrm{~kg} / \mathrm{m}^{3}\right)$ of $2.9 \mu \mathrm{m}$ diameter were used at a very low concentration in an aqueous solution $\left(\rho_{f}=1000 \mathrm{~kg} / \mathrm{m}^{3}\right)$. Therefore, the gravitational force, the Brownian force, and the forces due to multiparticle electrical interactions can be neglected according to a dimensional analysis. Consequently, the Langevin equation is simplified to

$$
m \frac{d \vec{u}_{p}}{d t}=\vec{F}_{D E P}-6 \pi \mu_{f} a \cdot\left(\vec{u}_{m}-\vec{u}_{p}\right)
$$

Solving this equation provides the particle velocity

$$
\vec{u}_{p}=\left(\frac{\vec{F}_{D E P}}{6 \pi \mu_{f} a}+\vec{u}_{m}\right) \cdot\left(1-e^{-\frac{6 \pi \mu_{f} a}{m} t}\right) \cong \frac{\vec{F}_{D E P}}{6 \pi \mu_{f} a}+\vec{u}_{m}
$$


The inertia term can be neglected because the relaxation frequency $f=\frac{6 \pi \mu_{f} a}{m} \sim 10^{7} \mathrm{~Hz}$ is considerably higher than the frequency of the applied electric field $\left(\sim 10^{5} \mathrm{~Hz}\right)$. Clearly, the competition between the DEP force and the viscous drag determines the velocity lag between the particle and the fluid. At equilibrium, both forces should balance each other. If the viscous drag is exceeded by the DEP driving force, the particles accelerate until a new equilibrium is established.

The dielectrophoretic particle motion perturbs an otherwise stationary fluid and generates a local flow field in the particle's vicinity, which can be described by Stokes' equation [21],

$$
\nabla^{2} \vec{V}=\frac{1}{\mu_{f}} \nabla p
$$

For simplicity, the torque on the particle due to stresses exerted by the surrounding fluid is not considered, and therefore the angular momentum does not play a role in the flow field.

The Stokes equation must be solved in conjunction with the continuity equation as well as the no-slip boundary condition at the surface of the particle,

$$
\nabla \cdot \vec{V}=0
$$

and at the surface of the particle,

$$
\vec{V}=\vec{u}_{p}
$$

The resulting velocity field is plotted in Figure 7, where the velocity is normalized with the particle velocity $u_{p}$. It is seen that, immediately around the particle, the fluid elements attain a velocity almost equal to $u_{p}$, as expected. However, the agitation the particle causes in the fluid extends well beyond its vicinity. The entire fluid domain in the plot is influenced, extending over an area of $20 a \times 20 a$, which is roughly 100 times larger than the particle size. The fluid in most of the domain reaches a velocity of at least $u_{p} / 10$. It is thus clear that a single particle can induce an appreciable flow field over a region considerably larger than its own size.

In colloidal suspensions where multiple particles are present, additional hydrodynamic interactions between neighboring translating particles could result in an intensification of the induced flow field. The extent of this kind of hydrodynamic interaction depends on many factors such as the particle shape and size, the interparticle distance, and the respective orientation of the particles. Consequently, the flow field induced by the collective motion of a group of particles will differ from that due to a single particle. In view of the difficulty in obtaining analytical solutions for a multiple-body problem, the method of reflections [21] is used; successive iterations are employed to solve the flow field to any degree of approximation by this method. The drag forces can be derived for a pair of identical particles separated by a distance $L$, as shown in Figure 8 for the simplest case of a multiple-body system,

$$
F_{\mu, x}=-\frac{6 \pi \mu_{f} a u_{p} \sin \alpha}{1+(3 / 4)(a / L)}
$$




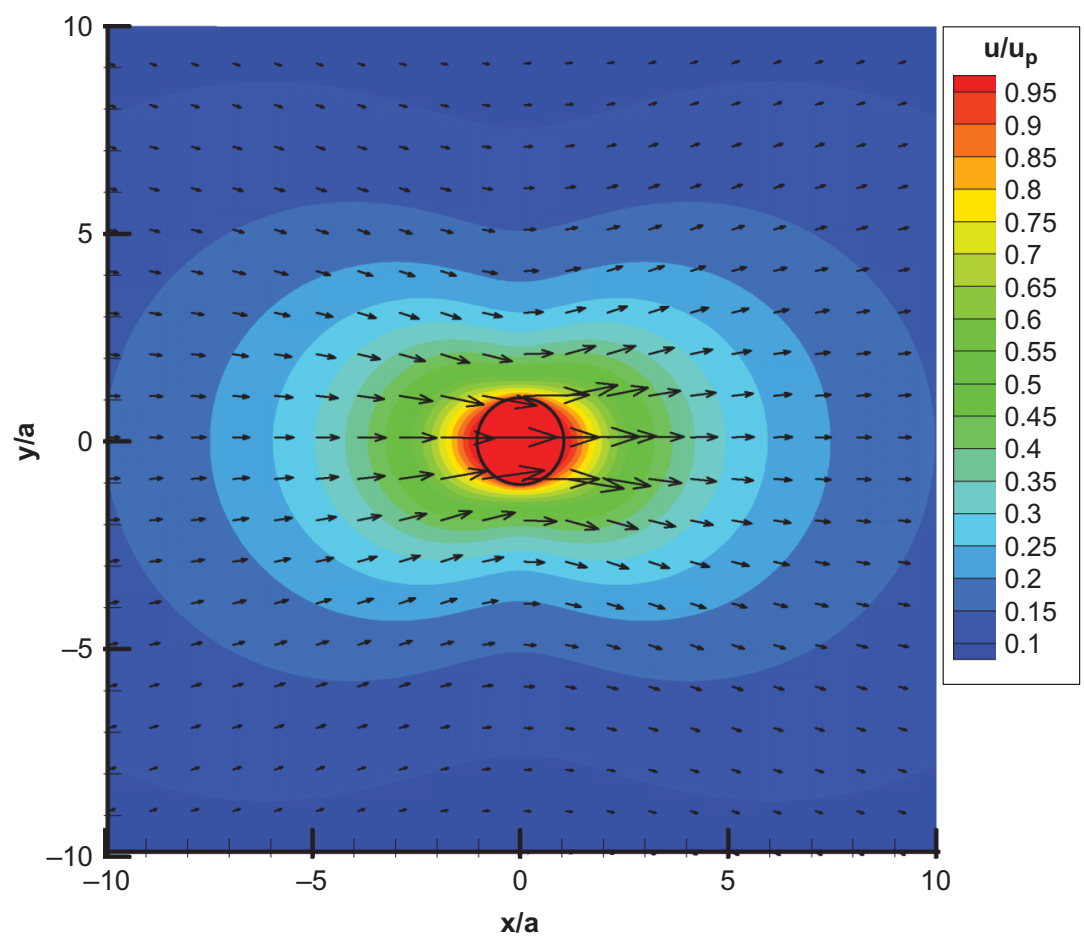

Figure 7 Solution of the velocity field around a translating particle. The circle designates the particle, which is translating from left to right. The magnitude of the velocity field is indicated by the color contours.

$$
F_{\mu, y}=-\frac{6 \pi \mu_{f} a u_{p} \cos \alpha}{1+(3 / 2)(a / L)}
$$

The particles in this case are considered to move with the same velocity along a direction at an angle $\alpha$ to the line joining their centers.

The equations reveal that the drag force experienced by each particle in the pair is strongly affected by the interparticle distance. If the two particles are very far apart $(L \rightarrow \infty)$, the particle-particle interaction can be neglected and the drag force reduces to the prediction from Stokes' drag law. As the interparticle distance decreases, the drag force decreases from the Stokes' drag law value, as indicated by the term in the denominator in Eqs. (15) and (16) This is because the motion of particle $a$ induces a flow velocity at the position of particle $b$, which helps to reduce the velocity lag between particle $b$ and its surrounding and leads to lower viscous drag on particle $b$, and vice versa. However, the DEP force on the particles is not affected by their relative positions. As stated earlier, the imbalance between the unaffected DEP force and the waning viscous drag will accelerate the particles to a higher velocity until a new equilibrium is reached. Consequently, the induced flow field is intensified.

Knowing the particle velocity and the drag force from Eqs. (8), (15), and (16), the flow field at the new equilibrium state can be deduced using the point-force approach [21]. The results are shown in Figure 9 for two specific situations. In Figure 9(a), the particles are 


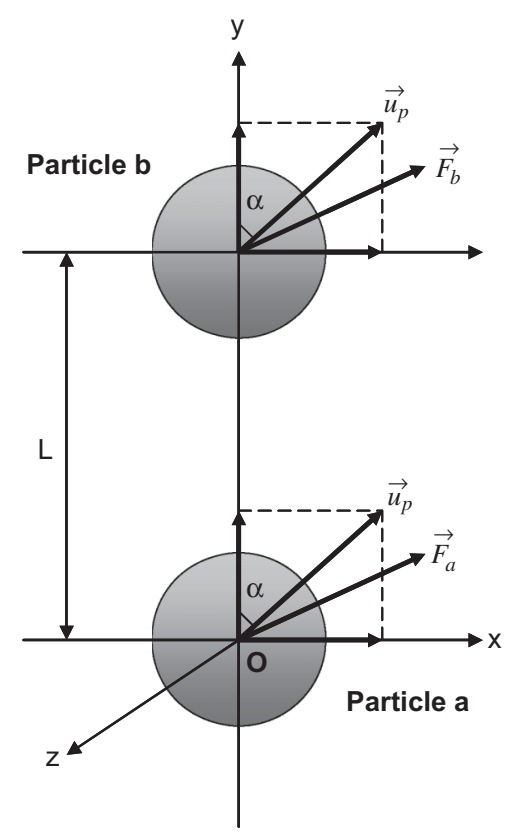

Figure 8 A simplified two-particle system. The two particles are identical in size at $r=a$ and separated from each other by a distance $L$. They move at the same velocity $\vec{u}_{p}$ with an angle $\alpha$ to the line joining their centers.

moving along the line joining their centers, that is, $\alpha=0$, and in Figure 9(b), the particles are moving perpendicular to the centerline, that is, $\alpha=90^{\circ}$. For both cases, the interparticle distance is decreased to explore the effect of this parameter on the induced flow field. Indeed, the flow fields are found to be enhanced, which can be attributed to two sources: the larger particle velocity due to the reduced viscous drag as a result of the particle-particle interaction and the superposition of flow fields due to the individual particles.

For a large number of particles, it is infeasible to study the hydrodynamic interaction and the flow field enhancement analytically. However, an estimate can be obtained by examining the superposition of flow fields due to individual particles in the suspension, which would provide an underestimate of the enhanced velocity field because the hydrodynamic interaction represented in Eqs. (15) and (16) is neglected. Figure 10 illustrates such flow fields induced by multiple-particle motion in suspensions. Because the interparticle distance is related to particle volume concentration, Figure 10 shows that the maximum velocity has increased to 3.3 times that of an individual particle at the higher particle concentration $(L=7.48 a)$, compared to an increase of 2.3 times at the lower particle concentration $(L=3.47 a)$.

The analysis in this section indicates the feasibility of generating substantial flow velocities based on hydrodynamic interactions between particles.

\section{NUMERICAL SIMULATION}

DEP and the induced flow field were analyzed above with simplified particle-fluid systems to elicit an understanding of the DEP driving force and the particle-fluid 

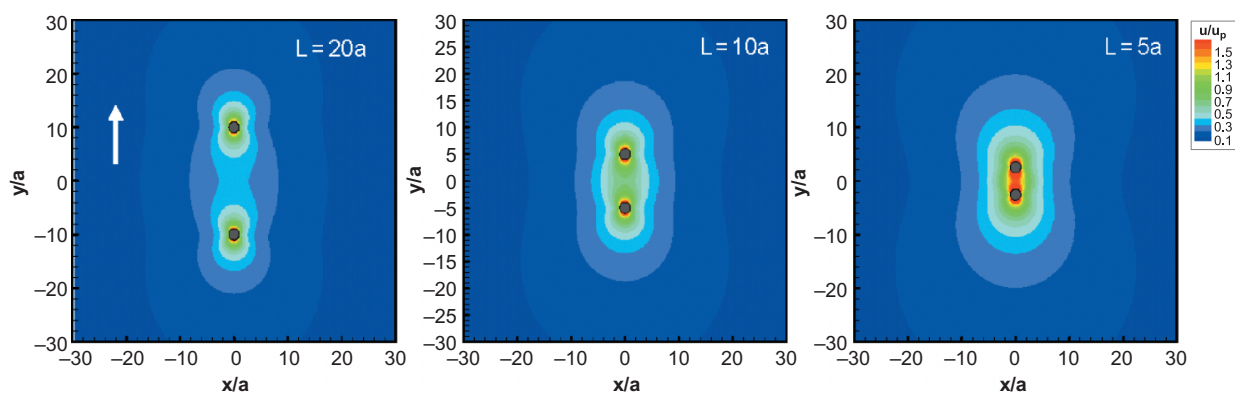

(a)
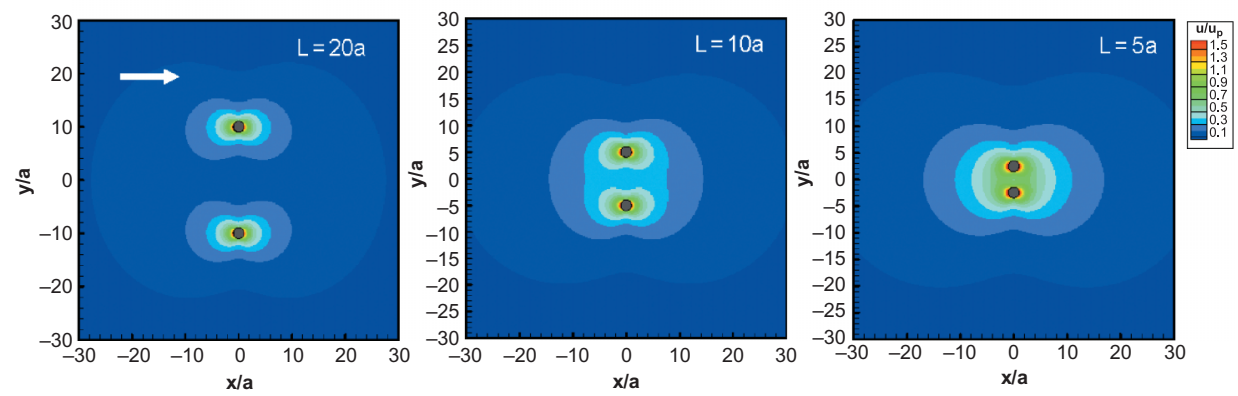

(b)

Figure 9 Enhancement of induced flow due to the hydrodynamic interaction between neighboring particles: (a) two particles moving toward each other and (b) two particles moving perpendicular to the line joining their centers. The interparticle distance is decreased from left to right from $20 a$ to $5 a$, leading to an intensified flow field.

interaction as a mechanism for microfluidic actuation. However, it is difficult to extend this analysis to general particle suspensions due to the complexity of solving a problem with the simultaneous presence of many particles. Hence, a numerical model is developed to study the flow physics for particle suspensions and to extract detailed information of the DEP-induced flow field.

The computational domain for the numerical model is shown in Figure 3. The electric and the flow fields are decoupled from each other and solved sequentially using a commercial software package, FLUENT [33]. The solution of the electric field has been described earlier and yields the DEP forces. The DEP force is computed for every point in space. However, only if a particle passes by a fluid element will there be a force acting on the fluid. Because the particles are present discretely in space, the DEP force is also dispersed in the fluid. However, there are ample particles in the suspension and their random passage in space makes their presence ergodic. As such, the DEP force, although actually acting on the discrete particles, can be treated as a continuous body force in the fluid by volume-averaging. In other words, the DEP force on one particle is averaged over the fluid volume surrounding the particle with the size of the averaging volume determined by the particle volume fraction. This DEP force is then introduced as a body force in the Navier-Stokes equations to solve for the induced flow field. By following this procedure, the complex solid-liquid two-phase flow problem is converted to a more straightforward single-phase fluid flow problem. 


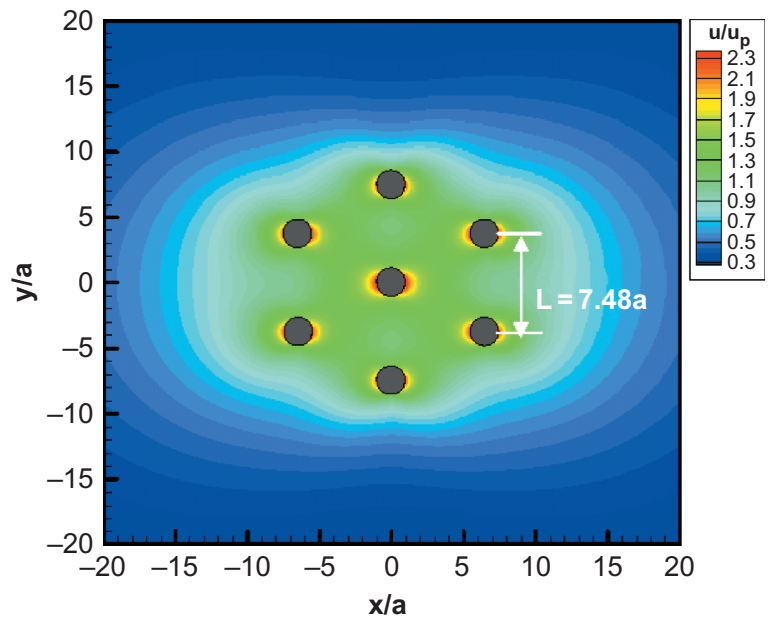

(a)

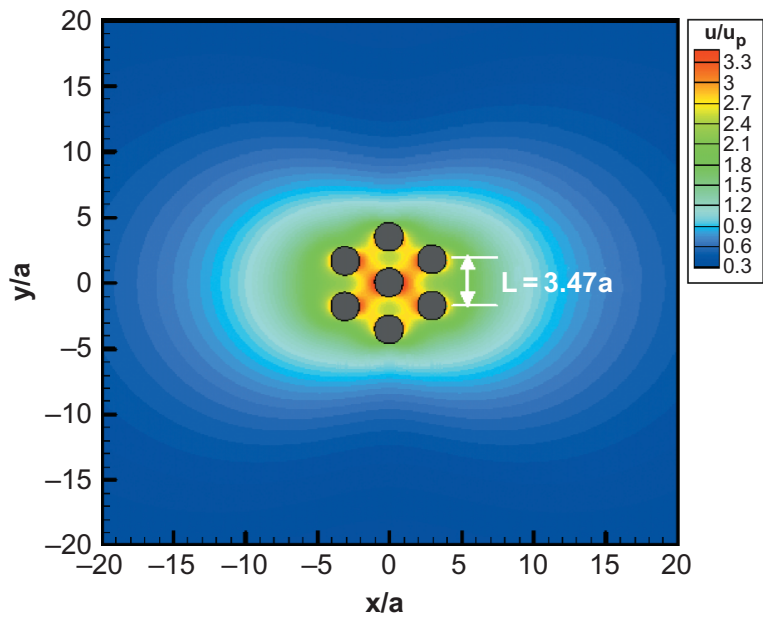

(b)

Figure 10 Velocity field enhancement due to increasing particle concentration: (a) $L=7.48 a$, and (b) $L=$ 3.47a. In this calculation, only superposition of the flow fields from individual particles is considered, which provides a conservative estimate of the degree to which the flow field is intensified.

The computational domain used for the flow field simulation is shown in Figure 3. Periodic velocity boundary conditions are specified at both ends of the domain along the $x$-direction, and no-slip boundary conditions are assumed for the top and bottom walls. The convective term is discretized using a first-order upwind scheme. The computational domain is discretized using a $600 \times 200(x-y)$ grid. Simulations with different grids showed a satisfactory grid independence for the results obtained with this mesh. The simulations are performed for 15 cases to study the effects of varying the frequency and voltage of the applied field on the induced flow field. The simulation matrix is shown in Table 1. The interparticle distance is maintained at $L=8 a$ for all cases, which corresponds to the actual spacing for a particle concentration of $\sim 1 \%$. For the selected frequencies, the particles experience both negative and traveling-wave DEP forces. 
Figure 11 shows the DEP-induced velocity field in the flow channel. Velocity profiles at various streamwise locations resemble the parabolic shape of pressuredriven flows. However, the profiles are asymmetric along the $y$-direction with appreciable distortions in regions right above the electrodes. Reverse flows also occur in the near-wall area, as indicated by the inset in the velocity contour plot. Velocity profiles of this type differ from other electrohydrodynamic flows, such as the plug profile observed in electroosmotic flows [10]. This difference is related to the traveling-wave DEP force shown in Figure 6, which demonstrates an almost constant driving force in the bulk fluid, similar to pressure-driven flows, except for regions near the electrodes.

Flow velocities at the midway location of the flow channel $(x=0.0003 \mathrm{~m})$ are plotted in Figure 12 for selected cases. For a given frequency, the velocity increases with increasing applied voltage as a result of the enhanced driving forces. However, modulating the frequency of the electric field appears to be a far more effective way to increase the flow velocity. For instance, the induced velocities at $10 \mathrm{kHz}$ even at lower voltages (22 and $22.8 \mathrm{~V}$ ) exceed that at the maximum voltage $(50 \mathrm{~V})$ at $100 \mathrm{kHz}$.

Table 1 Numerical simulation matrix

\begin{tabular}{llcc}
\hline$f(\mathrm{kHz})$ & $\mathrm{Re}\left[f_{\mathrm{CM}}\right]$ & $\operatorname{Im}\left[f_{\mathrm{CM}}\right]$ & $V($ Volt $)$ \\
\hline 10 & -0.008 & -0.562 & 10 \\
10 & -0.008 & -0.562 & 15.6 \\
10 & -0.008 & -0.562 & 22 \\
10 & -0.008 & -0.562 & 28.6 \\
10 & -0.008 & -0.562 & 50 \\
50 & -0.451 & -0.162 & 10 \\
50 & -0.451 & -0.162 & 15.6 \\
50 & -0.451 & -0.162 & 22 \\
50 & -0.451 & -0.162 & 28.6 \\
50 & -0.451 & -0.162 & 50 \\
100 & -0.468 & -0.0823 & 10 \\
100 & -0.468 & -0.0823 & 15.6 \\
100 & -0.468 & -0.0823 & 22 \\
100 & -0.468 & -0.0823 & 28.6 \\
100 & -0.468 & -0.0823 & 50 \\
\hline
\end{tabular}

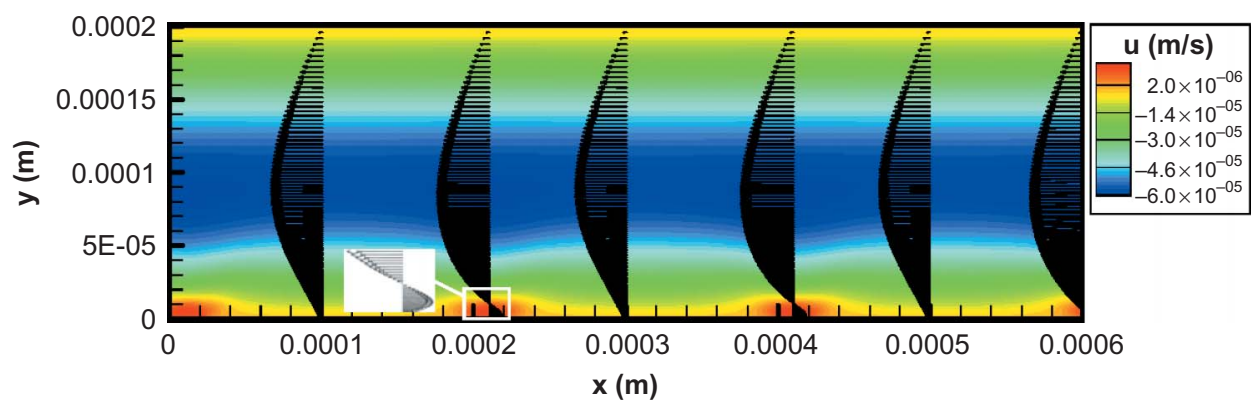

Figure 11 DEP-induced velocity profiles at various streamwise locations. The frequency of the applied signal is $f=10 \mathrm{kHz}$ and the voltage is $V_{0}=28.6 \mathrm{~V}$. 


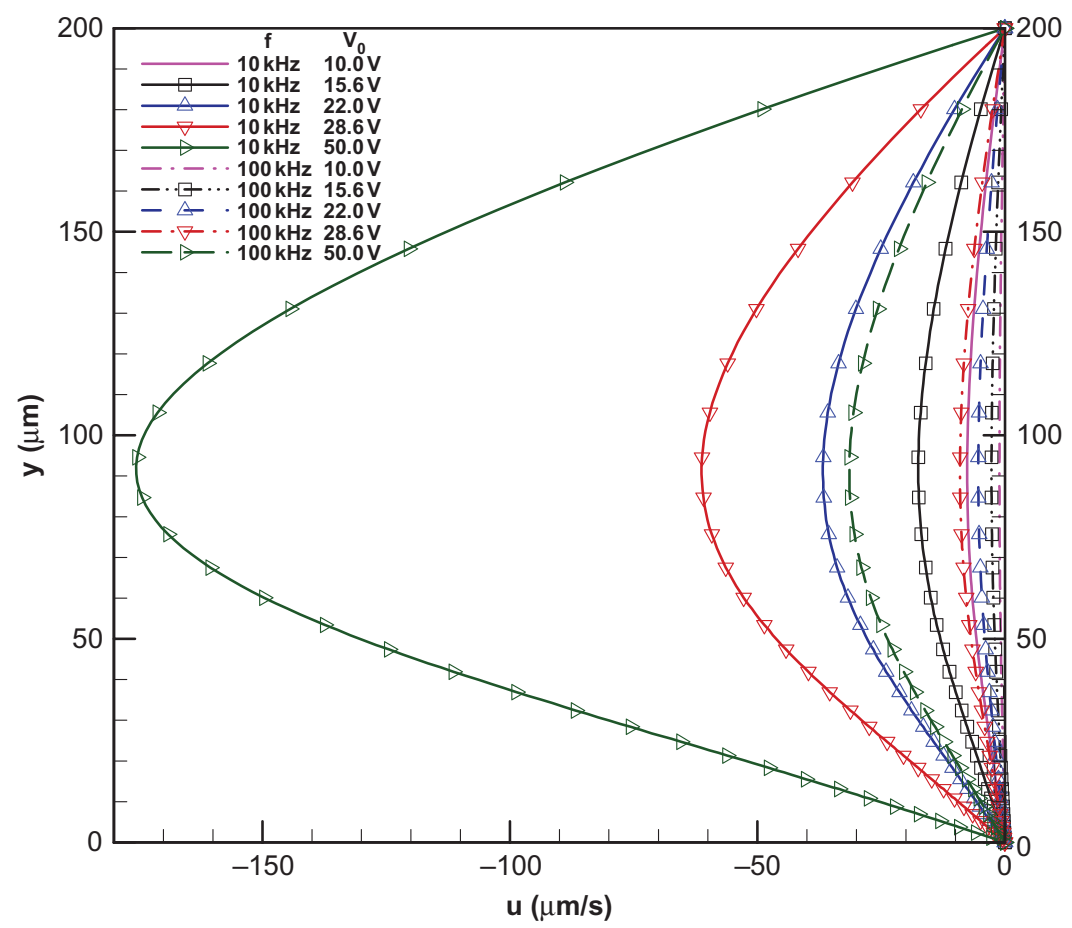

Figure 12 Velocity profile at the midway location in the direction of flow $(x=0.0003 \mathrm{~m})$ for selected conditions.

\section{EXPERIMENTS}

To demonstrate the proposed DEP-induced microfluidic pumping concept, a prototype device was designed and fabricated. The device consists of an array of interdigitated microelectrodes fabricated using photolithography. The microelectrodes are made of a layer of 100-nm-thick gold that is e-beam evaporated onto a nonoxidized silicon wafer. The array contains 10 parallel thin-bar microelectrodes, $20 \mu \mathrm{m}$ wide each and separated by $180 \mu \mathrm{m}$ gaps. The rather large gap was chosen to reduce electrical leakage between electrodes and to alleviate electrothermal effects caused by Joule heating. A layer of Parylene C (thickness $500 \mathrm{~nm}$ ) was deposited over the electrode array to avoid electrolysis and corrosion of the electrodes when the device is in contact with the particle suspensions. The prototype DEP device is shown in Figure 13(a). A flow channel is constructed by placing a $500-\mu \mathrm{m}$-thick Pyrex glass slide over two 200- $\mu \mathrm{m}$-thick spacers on either side of the device, which are sealed with epoxy as shown in Figure 13(b). The particle suspensions are prepared by thoroughly mixing polystyrene microparticles of $2.9 \mu \mathrm{m}$ diameter (Duke Scientific, CA) with deionized water using a Thermolyne stirrer. The volume fraction is estimated to be $1 \%$.

In the experiments, the wire-bonded DEP device is mounted on a printed circuit board and the electrodes connected to an AC voltage of frequency $f$, as shown in Figure 14. The applied electric signals are controlled by a pulse generator (Berkeley Nucleonics Model 565, CA) and a custom-built timing circuit. The applied voltage ranges from 10 to $30 \mathrm{~V}$, with frequencies ranging from 1 to $1000 \mathrm{kHz}$. A digital 


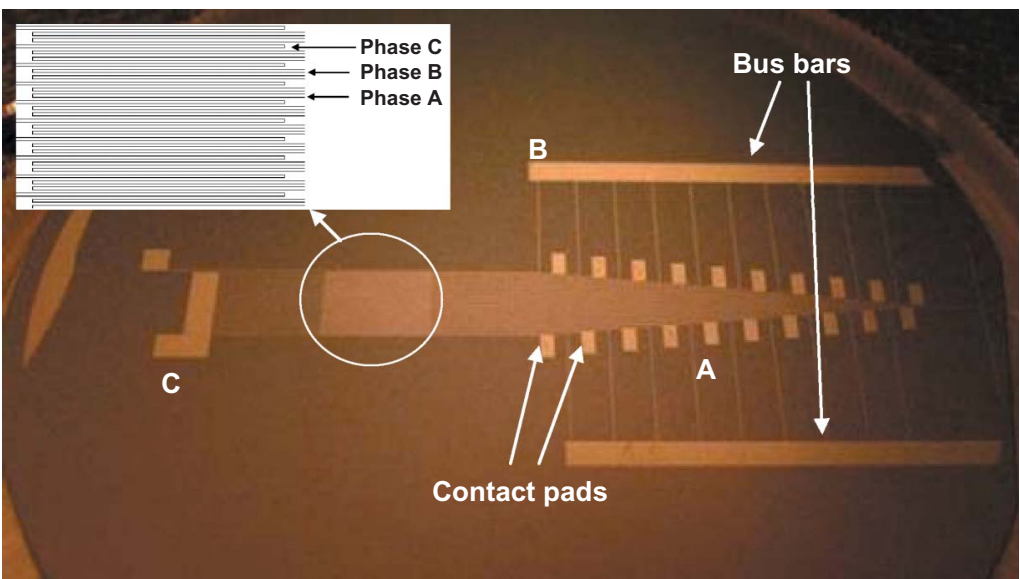

(a)

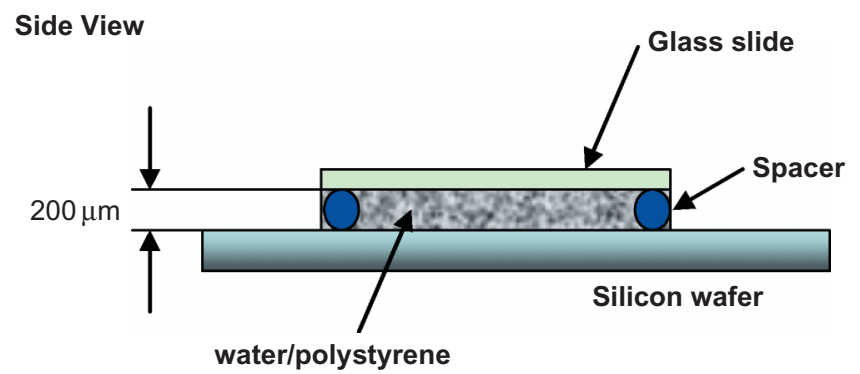

(b)

Figure 13 (a) The prototype DEP device and (b) cross-sectional view of the DEP micropumping test piece.

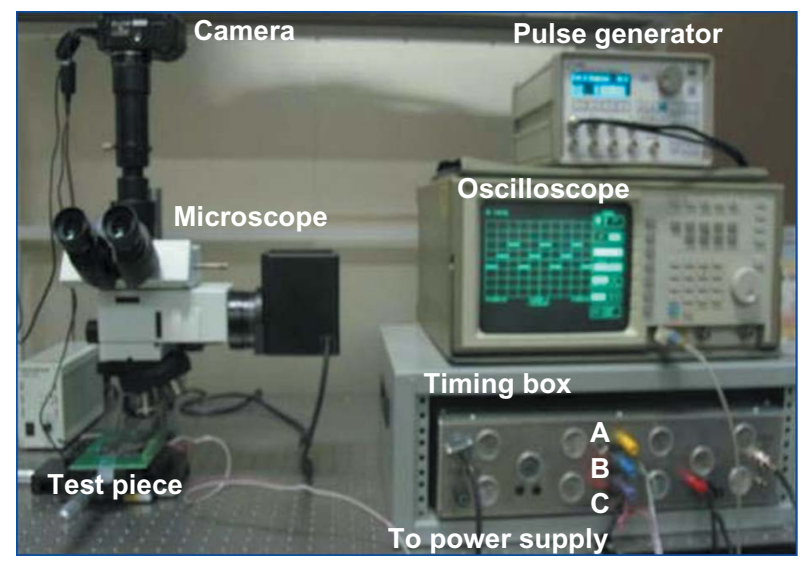

Figure 14 DEP micropumping experimental apparatus. 
oscilloscope (Tektronix TDS 3032B, OR) is used to monitor the frequency and waveform of the applied signals during the experiments. The particle motion is recorded with a CCD camera (Olympus C5060) under an Olympus BXFM microscope. An Olympus LMPLFL $20 \times$ objective lens $($ N.A. $=0.4$, working distance $=12 \mathrm{~mm}$ ) is used for the measurement.

Figure 15(a) shows the random dispersion of particles before application of the electric field. The particles oscillate a little around their equilibrium positions due to Brownian motion. Once a low-frequency signal (below $1 \mathrm{kHz}$ ) is applied, the particles collect at the edge of the electrode, as shown in Figure 15(b), designating the occurrence of positive DEP. Upon increasing the frequency to $100 \mathrm{kHz}$, a negative DEP force causes the particles to be repelled from the electrode to the gap region, as illustrated in Figure 15(c). If the frequency falls in the effective twDEP range $(10-100 \mathrm{kHz})$, the particles experience traveling-wave DEP forces and travel in the transverse plane parallel to the microelectrode array. Positions of the particles at consecutive time instants under this condition were recorded at a $15 \mathrm{fps}$ frame rate. In the measurements, the microscope was adjusted to focus at a distance from the wall where the particle velocity is visualized to reach its peak. The translational motion of individual particles is clearly illustrated in Figure 16.

Microparticle image velocimetry ( $\mu \mathrm{PIV}$ ) was used in conjunction with the images to obtain quantitative measurements of the spatially resolved velocity field [37]. The measurement uncertainty in the particle velocity was estimated to be $5.14 \mu \mathrm{m} / \mathrm{s}$.

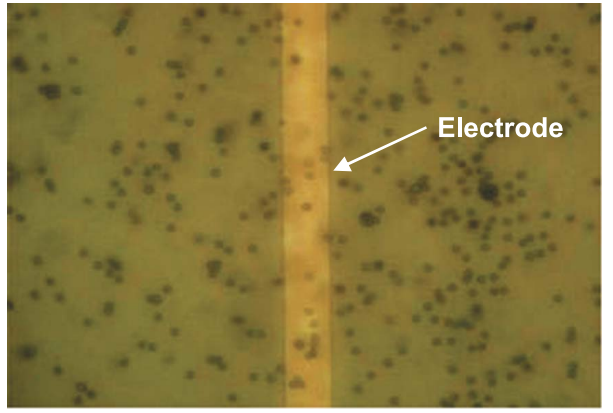

(a)

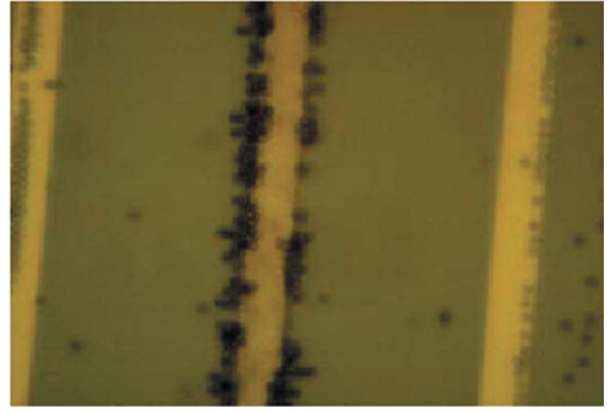

(b)

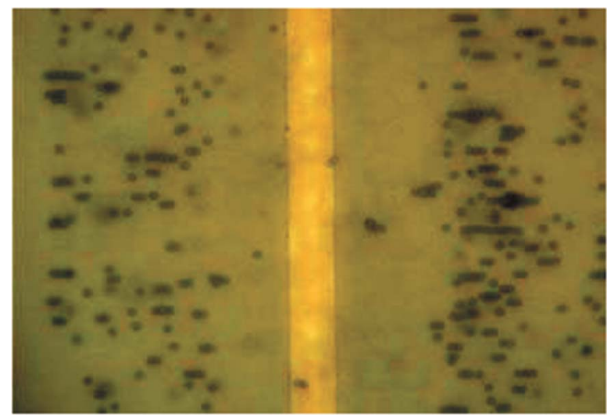

(c)

Figure 15 (a) Random dispersion of microparticles before applying electric field; (b) positive DEP occurs at low frequencies and the particles collect on the microelectrodes; and (c) negative DEP occurs at higher frequencies, and the particles are repelled from the microelectrodes. 

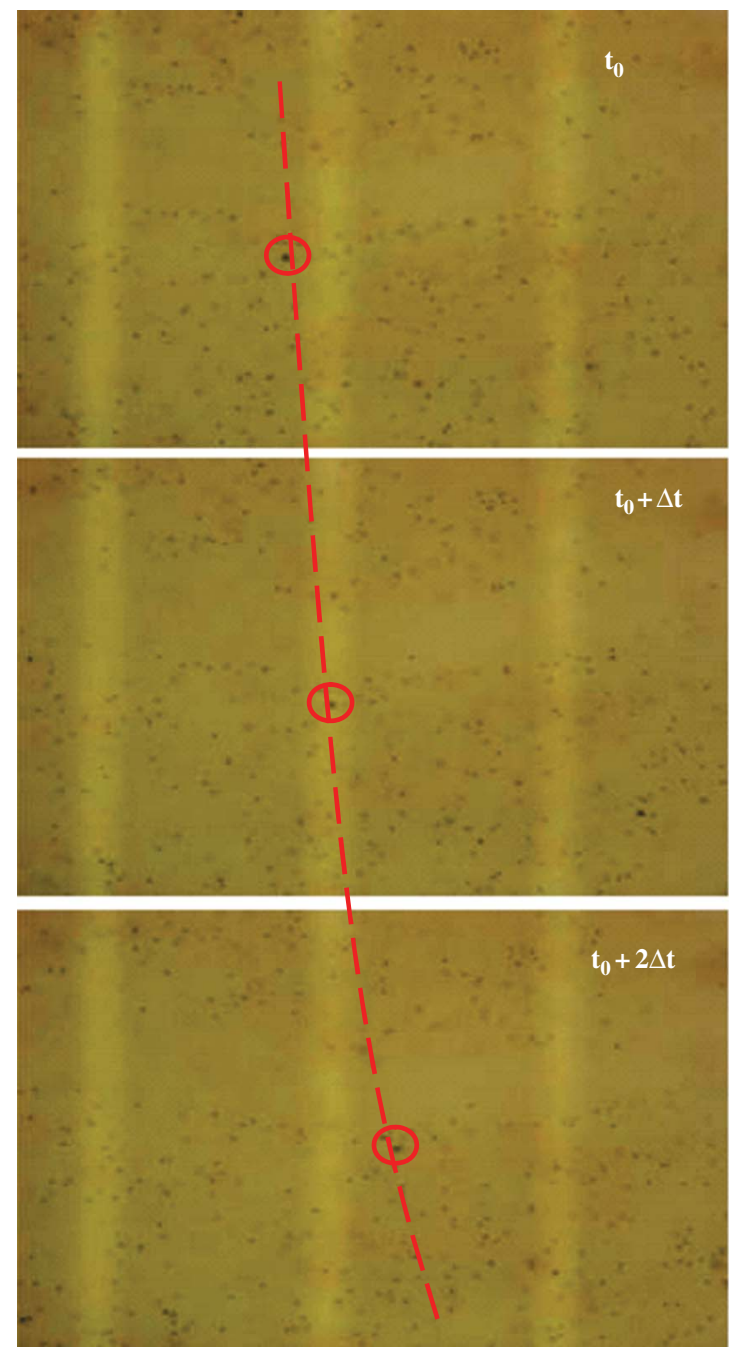

Figure 16 Particle motion with twDEP. Consecutive exposures of a particle are tracked in the three photographs.

Figure 17 shows a sample result of the measured particle velocity field. It can be seen that the velocity field is nearly uniform within the measurement plane. From Eq. (11), it is expected that a velocity lag exists between the particle and the surrounding fluid at equilibrium, which must be considered in deducing the flow field from the $\mu$ PIV measurements. Note that the traveling-wave DEP component is the driving force for the observed particle motion. Therefore, the velocity lag can be estimated from

$$
\left|\vec{u}_{p}-\vec{u}_{m}\right| \cong\left|\frac{\vec{F}_{t w D E P}}{6 \pi \mu_{f} a}\right|
$$




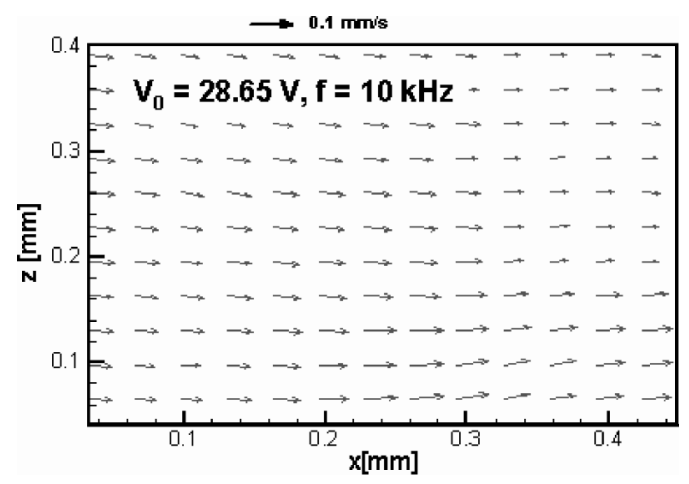

Figure $17 \mu$ PIV measurement of the DEP-induced particle velocity field.

where

$$
\vec{F}_{t w D E P}=2 \pi a^{3} \varepsilon_{m} \operatorname{Im}\left[f_{C M}\right]\left(E_{x}^{2} \nabla \varphi_{x}+E_{y}^{2} \nabla \varphi_{y}+E_{z}^{2} \nabla \varphi_{z}\right)
$$

For instance, for the experimental conditions associated with the $\mu$ PIV measurement in Figure $17\left(V_{0}=28.6 \mathrm{~V}\right.$ and $\left.f=10 \mathrm{kHz}\right)$, the velocity lag is approximately $5.2 \mu \mathrm{m} / \mathrm{s}$ so that a flow velocity of $47.1 \mu \mathrm{m} / \mathrm{s}$ may be deduced. Upon changing the focal plane of the microscope objective, it was observed that the velocity profile qualitatively followed the parabolic distribution shown in Figure 12. Given the nearuniform twDEP force and the distance from the electrodes, this is only possible through hydrodynamic interactions between the particles and the fluid. The average maximum flow velocities extracted from the $\mu \mathrm{PIV}$ measurements are plotted in Figure 18. Comparison of the experimental data with the numerical results shows

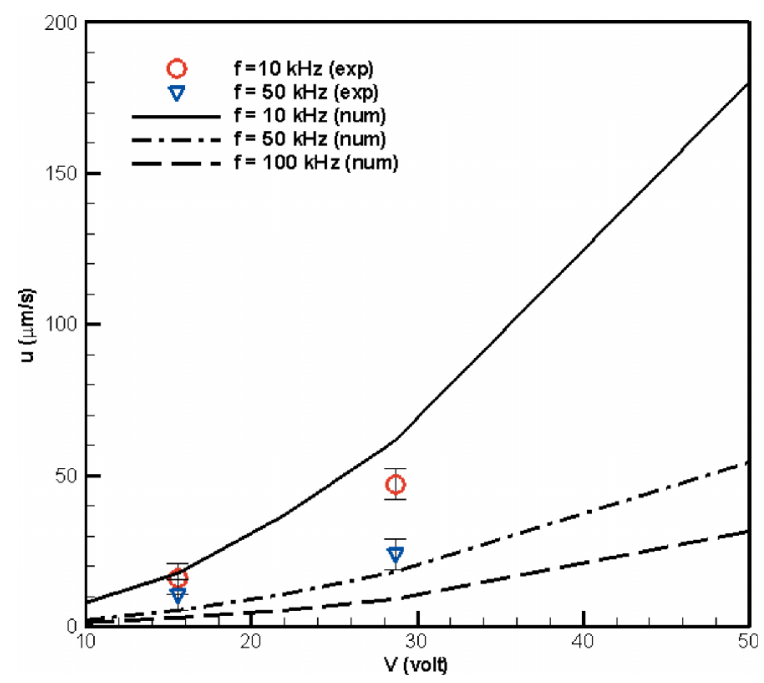

Figure 18 Comparison of the measured flow velocity with the numerical results. The measurement uncertainty in the flow velocity is $5.14 \mu \mathrm{m} / \mathrm{s}$. 
satisfactory agreement. In addition, the numerical results imply that the flow velocity could be tripled to $180 \mu \mathrm{m} / \mathrm{s}$ upon doubling the voltage to $50 \mathrm{~V}$ at $10 \mathrm{kHz}$. For optimized electrode design and appropriate selection of particle concentration, it is expected that the induced flow field can be significantly enhanced. It is noted that other electrokinetic phenomena such as the induced charge electroosmosis (ICEO) [38] and AC electroosmosis (ACEO) [39] can occur upon the application of AC electric fields, which may also induce particle motion and fluid flow. However, these phenomena only persist over a certain band of frequencies $(10 \mathrm{~Hz}<f<10 \mathrm{kHz})$ that is quite different from the frequency range in the present study $(10 \mathrm{kHz}<f<100 \mathrm{kHz})$. It is reasonable to conclude that the observed fluid pumping in this work is solely due to the twDEP action.

\section{PERSPECTIVES}

In the DEP-induced microfluidic pumping studied in this work, a number of approximations are made in the analysis to render the problem tractable. For instance, rotational motion and Brownian dynamics are neglected, and the effects of the boundary wall and multipole interactions are ignored in considering the collective motion of particle suspensions. One important measure of a pumping approach is its thermodynamic efficiency, defined as the useful pressure work divided by total power consumption [40]. Using a scaling analysis, the thermodynamic efficiency of the prototype DEP micropump is estimated to be rather low $\left(\sim 10^{-6} \%\right)$, mainly due to the relatively large electrode width and spacing used in fabrication of the prototype. The same scaling analysis reveals that the geometry of the microelectrode array can be optimized together with the particle concentration to improve the pumping efficiency to $1 \%$, a typical value for electrokinetic micropumps [40], and to produce higher flow velocity for engineering applications.

In ongoing work, the potential of the proposed technique is being explored further by extending it to the pumping of nanofluids in microfluidic devices. Nanofluids are novel engineered colloids that exhibit superior thermal transport properties owing to the presence of the dispersed nanoparticles [41]. However, the concomitant increase in viscosity poses a challenge for circulating nanofluids in microfluidic systems with traditional pumping methods [42]. The DEP-induced micropumping technique will be used to actuate and control nanofluids such that the nanoparticles themselves act as the fluid mover while, at the same time, contributing to enhancement in thermal transport.

\section{CONCLUSIONS}

A microfluidic pumping scheme based on traveling-wave dielectrophoresis (twDEP) is proposed for generating flow in situ in microfluidic devices. This technique utilizes the dielectrophoretic motion of small particles and their hydrodynamic interactions with the surrounding fluid to achieve a self-contained microscale fluid delivery system with no moving parts. Fundamental aspects of this technique, such as the traveling-wave electric field, DEP force, particle dynamics, and particle-fluid hydrodynamic interactions, are discussed in detail. A numerical model is formulated to simulate the DEP-induced flow field. A prototype DEP micropumping device is fabricated and characterized with polystyrene-water suspensions. This technique provides a powerful, versatile tool for actuating colloidal suspensions in microfluidic devices. 


\section{REFERENCES}

1. J.Z. Hilt and N.A. Peppas, Microfabricated Drug Delivery Devices, International Journal of Pharmaceutics, vol. 306, pp. 15-23, 2005.

2. H.R. Sahoo, J.G. Kralj, and K.F. Jensen, Multistep Continuous-Flow Microchemical Synthesis Involving Multiple Reactions and Separations, Angewandte Chemie International Edition, vol. 46, pp. 5704-5708, 2007.

3. D. Erickson and D. Li, Integrated Microfluidic Devices, Analytica Chimica Acta, vol. 507, pp. 11-26, 2004.

4. V. Singhal and S.V. Garimella, Induction Electrohydrodynamics Micropump for High Heat Flux Cooling, Sensors and Actuators A, vol. 134, pp. 650-659, 2007.

5. V. Singhal, S.V. Garimella, and A. Raman, Microscale Pumping Technologies for Microchannel Cooling Systems, Applied Mechanics Reviews, vol. 57, pp. 191-221, 2004.

6. B. Iverson and S.V. Garimella, Recent Advances in Microscale Pumping Technologies: A Review and Evaluation, Microfluidics and Nanofluidics, vol. 5, pp. 1613-4982, 2008.

7. V. Singhal and S.V. Garimella, A Novel Valveless Micropump with Electrohydrodynamic Enhancement for High Heat Flux Cooling, IEEE Transactions on Advanced Packaging, vol. 28, pp. 216-230, 2005.

8. W. Zhang, T.S. Fisher, and S.V. Garimella, Simulation of Ion Generation and Breakdown in Atmospheric Air, Journal of Applied Physics, vol. 96, pp. 6066-6072, 2004.

9. D. Go, S.V. Garimella, T.S. Fisher, and R.K. Mongia, Ionic Winds for Locally Enhanced Cooling, Journal of Applied Physics, vol. 102, 053302, 2007.

10. S. Zeng, C.H. Chen, J.C. Mikkelsen, and J.G. Santiago, Fabrication and Characterization of Electroosmotic Micropumps, Sensors and Actuators B, vol. 79, pp. 107-114, 2001.

11. A. Ramos, H. Morgan, N.G. Green, A. Gonzalez, and A. Castellanos, Pumping of Liquids with Traveling-Wave Electroosmosis, Journal of Applied Physics, vol. 97, 084906, 2005.

12. B.P. Cahill, L.J. Heyderman, J. Gobrecht, and A. Stemmer, Electro-Osmotic Pumping on Application of Phase-Shifted Signals to Interdigitated Electrodes, Sensors and Actuators B, vol. 110, pp. 157-163, 2005.

13. H. Morgan, M.P. Hughes, and N.G. Green, Separation of Submicron Bioparticles by Dielectrophoresis, Biophysics Journal, vol. 77, pp. 516-525, 1999.

14. J.A. Eastman, S.R. Phillpot, S.U.S. Choi, and P. Keblinski, Thermal Transport in Nanofluids, Annual Review of Materials Research, vol. 34, pp. 219-246, 2004.

15. H.A. Pohl, The Motion and Precipitation of Suspensoids in Divergent Electric Fields, Journal of Applied Physics, vol. 22, pp. 869-871, 1951.

16. P.R.C. Gascoyne and J. Vykoukal, Particle Separation by Dielectrophoresis, Electrophoresis, vol. 23, pp. 1973-1983, 2002.

17. M.P. Hughes and H. Morgan, Dielectrophoretic Trapping of Single Sub-micrometre Scale Bioparticles, Journal of Physics D, vol. 31, pp. 2205-2210, 1998.

18. S. Fiedler, S.G. Shirley, T. Schnelle, and G. Fuhr, Dielectrophoretic Sorting of Particles and Cells in a Microsystem, Analytical Chemistry, vol. 70, pp. 1909-1915, 1998.

19. J. Suehiro and R. Pethig, The Dielectrophoretic Movement and Positioning of a Biological Cell Using a Three-Dimensional Grid Electrode System, Journal of Physics D, vol. 31, pp. 3298-3305, 1998.

20. W.B. Russel, D.A. Saville, and W.R. Schowalter, Colloidal Dispersions, Cambridge University Press, Cambridge, UK, 1989.

21. J. Happel and H. Brenner, Low Reynolds Number Hydrodynamics with Special Applications to Particulate Media, Springer, New York, 1983.

22. T.B. Jones, Electromechanics of Particles, Cambridge University Press, Cambridge, UK, 1995.

23. H.A. Pohl, Dielectrophoresis, Cambridge University Press, Cambridge, UK, 1978. 
24. P.R.C. Gascoyne, Y. Huang, R. Pethig, J. Vykoukal, and F.F. Becker, Dielectrophoretic Separation of Mammalian Cells Studied by Computerized Image Analysis, Measurement Science and Technology, vol. 3, pp. 439-445, 1992.

25. H. Morgan and N.G. Green, AC Electrokinetics: Colloids and Nanoparticles, Research Studies Press, Philadelphia, 2003.

26. H. Morgan, A.G. Izquierdo, D. Bakewell, N.G. Green, and A. Ramos, The Dielectrophoretic and Traveling Wave Forces Generated by Interdigitated Electrode Arrays: Analytical Solution Using Fourier Series, Journal of Physics D, vol. 34, pp. 1553-1561, 2001.

27. R. Hagedorn, G. Fuhr, T. Muller, and J. Gimsa, Traveling-Wave Dielectrophoresis of Microparticles, Electrophoresis, vol. 13, pp. 49-54, 1992.

28. X.J. Wang, X.B. Wang, F.F. Becker, and P.R.C. Gascoyne, A Theoretical Method of Electrical Field Analysis for Dielectrophoretic Electrode Arrays Using Green's Theorem, Journal of Physics D, vol. 29, pp. 1649-1660, 1996.

29. D.S. Clague and E.K. Wheeler, Dielectrophoretic Manipulation of Macromolecules: The Electric Field, Physical Review E, vol. 64, 026605, 2001.

30. X.B. Wang, Y. Huang, J.P.H. Burt, G.H. Markx, and P. Pethig, Selective Dielectrophoretic Confinement of Bioparticles in Potential Energy Wells, Journal of Physics D, vol. 26, pp. $1278-1285,1993$.

31. M. Garcia and D.S. Clague, The 2D Electric Field above a Planar Sequence of Independent Strip Electrodes, Journal of Physics D, vol. 33, pp. 1747-1755, 1999.

32. N.G. Green, A. Ramos, and H. Morgan, Numerical Solution of the Dielectrophoretic and Travelling Wave Forces for Interdigitated Electrode Arrays Using the Finite Element Method, Journal of Electrostatics, vol. 56, pp. 235-254, 2002.

33. FLUENT 6 User's Guide, Lebanon, NH, Fluent Inc., 2000.

34. D.E. Chang, S. Loire, and I. Mezic, Closed-Form Solution in the Electrical Field Analysis for Dielectrophoretic and Traveling Wave Interdigitated Electrode Arrays, Journal of Physics D, vol. 36, pp. 3073-3078, 2003.

35. M. Llamas, V. Ginger, and S. Sancho, The Dynamic Evolution of Cell Chaining in a Biological Suspension Induced by an Electrical Field, Journal of Physics D, vol. 31, pp. 3160-3167, 1998.

36. D.J. Klingenberg, F. Van Swol, and C.F. Zukoski, Dynamic Simulation of Electrorheological Suspensions, Journal of Chemical Physics, vol. 91, pp. 7888-7895, 1989.

37. D. Liu, S.V. Garimella, and S.T. Wereley, Infrared Micro-Particle Image Velocimetry in Silicon-Based Microdevices, Experiments in Fluids, vol. 38, pp. 385-392, 2005.

38. T.M. Squires and M.Z. Bazant, Induced-Charge Electro-Osmosis, Journal of Fluid Mechanics, vol. 509, pp. 217-252, 2004.

39. A. Ramos, H. Morgan, N.G. Green, and A. Castellanos, AC Electric-Field-Induced Fluid Flow in Microelectrodes, Journal of Colloid and Interface Science, vol. 217, pp. 420-422, 1999.

40. C.H. Chen and J.G. Santiago, A Planar Electroosmotic Micropump, Journal of Microelectromechanical Systems, vol. 11, pp. 672-683, 2002.

41. J. Eastman, S.U.S. Choi, S. Li, W. Yu, and L.J. Thompson, Anomalously Increased Effective Thermal Conductivities of Ethylene Glycol-Based Nanofluids Containing Copper Nanoparticles, Applied Physics Letters, vol. 78, pp. 718-720, 2001.

42. B.C. Pak and Y. Cho, Hydrodynamic and Heat Transfer Study of Dispersed Fluids with Submicron Metallic Oxide Particles, Experimental Heat Transfer, vol. 11, pp. 151-170, 1998. 